\title{
ORDER ALGEBRAS
}

\author{
BY ROBERT L. DAVIS
}

Communicated by Gian-Carlo Rota, July 29, 1969

A partially ordered set $P$ in which every pair of elements has a greatest lower bound is a semigroup, with $p q=p \wedge q$, and hence is naturally associated with a semigroup algebra $Z[P]$ over the integers. For finite $P$ Solomon has given [3] a marvelously ingenious construction of an analogous sort of algebra even when $P$ is not a semilattice and so cannot be made into a semigroup. Semigroup algebras and Solomon's "Möbius algebras" have applications in combinatorial problems involving the underlying orders.

Now in a recent study [2] of valuations and Euler characteristics on lattices Rota introduced an ostensibly quite different sort of algebra he called a "valuation ring" which, rather surprisingly, plays a role like that of a semigroup algebra. More surprising, in view of their entirely different genesis and description, is that Rota's valuation ring can be shown to include Solomon's Möbius algebra as a special case.

Rota's construction, when used to associate such an algebra to a partial order $P$ (which is only one outgrowth of his inquiry), leads in stages through several different structures. The results implicitly provide a recursive procedure for computing products in the valuation ring $V(P)$, but give no direct formula. Solomon, on the other hand, defined his Möbius algebra by giving an explicit, if rather complicated, formula to express products of elements of $P$ as linear combinations of $P$-elements. The purpose of this note is to determine from Rota's construction an explicit formula for products in $V(P)$ which depends only on the order structure of $P$. This will show at once that Rota's construction includes Solomon's, and it can be recast in a particularly simple form that clarifies further consequences and applications.

AMS Subject Classifications. Primary 2090, 2095; Secondary 0525, 0620, 4620.

Key Words and Phrases. Order algebra, semigroup algebra, valuation ring, convolution algebra, Möbius algebra, incidence algebra, semicharacter, convolution transform. 
1. The Rota construction. Let $L=\{S, T, \cdots\}$ be any distributive lattice under $\cup$ and $\cap$, made into a semigroup by setting $S T=S \cap T$. In the semigroup algebra $K[L]$ over a commutative ring $K$ the submodule $Q$ generated by all $S+T-S T-S \cup T$ with $S$ and $T$ in $L$ is an ideal. Since valuations on $L$ are just those functionals which are identically zero on $Q$, Rota calls the quotient $K[L] / Q$ the valuation ring $V(L, K)$. The special case of interest in this note has $L$ the lattice of "order ideals" of a partial order $(P, \leqq)$ and $K=Z$, the ring of integers.

Let $P$ be such that every cone $C_{p}=\{q \in P: q \leqq p\}$ is finite and define $L$ to be the ring of sets generated by all cones, with $\varnothing$ added. Then $L$ is a distributive lattice whose finite elements admit the convenient height function, ht $S=|S|$ (number of elements in $S$ ). The quotient $Z[L] / Q$ may in this case, because of its likeness to the semigroup algebra of a semigroup, be called the order algebra, $V(P)$, of $P$.

Identifying elements of $L$ with their images in $V$, Rota extends the identity defining $Q$ to give a general inclusion-exclusion formula that expresses any finite union of lattice elements as a linear combination:

$$
S_{1} \cup \cdots \cup S_{r}=\sum_{i=1}^{r} S_{i}-\sum_{i<j} S_{i} S_{j}+\sum_{i<j<k} S_{i} S_{j} S_{k}-\cdots .
$$

LeMma 1. Any $S$ of finite height in $L$ is a well-defined linear combination, $S=\sum_{p \in P} \phi_{S}(p) C_{p}$, of cones contained in $S$ : that is $\phi_{S}(p)=0$ unless $C_{p} \subset S$.

The proof is by induction on the height of $S$. If ht $S=0$ then $S=\varnothing$ and this is $T+T-T T-T \cup T=0$.

Any other element of finite height in $L$ is either a cone or a finite union of join irreducibles (i.e. cones) of finite height. Now assume the lemma for elements of height $<h$ and suppose $h t S=h$. If $S$ is not itself a cone it must be an irredundant union, $S=C_{p_{1}} \cup \ldots \cup C_{p_{r}}$, of the maximal cones contained in $S$. By $(*), S=\sum C_{p_{i}}-\sum C_{p_{i}} C_{p_{j}}$ $+\cdots$, where each term $C_{p_{1}} \cdots C_{p_{k}}$ on the right has height $<h$ and hence, by induction, is a well-defined linear combination of cones $C_{q}$ contained in it, and thus in each $C_{p_{i}}$. Then $S$ is the well-defined linear combination gotten by adding all such terms, and furthermore each $C_{q} \subset C_{p_{i}} \subset S$.

(If $S$ is written as a nonirredundant union of cones, which can only be done by using all the maximal $C_{p_{i}}$ in $S$ and other cones $C_{r}$ contained within some of them, it is easy to show that the added contribution from the $C_{r}$ 's amounts to zero.)

Thus $V$ essentially consists of all linear combinations of cones and 
its multiplication can be taken to define a (commutative $Z$-algebra) product among $P$-elements, say $\circ$, by the rule: $x \circ y=\sum_{p \in P} \phi_{x y}(p) \cdot p$ if and only if $C_{x} C_{y}=\sum_{p \in P} \phi_{x y}(p) C_{p}$. This way of writing the $V(P)$ product brings out the analogy with semigroup algebras; of course, $V(P)$ is the integral semigroup algebra on $P$ if and only if $P$ is a semilattice.

2. Explicit formula for the product. Rota's procedures show how to compute such products by working upward from minimal elements, but provide no direct way to determine $C_{x} C_{y}$. With only these recursive techniques to build on it is natural to seek an explicit formula by repeated use of induction in the identity $(*)$.

Suppose now that the maximal cones in a given $C_{x} C_{y}=C_{x} \cap C_{y}$ are $C_{p_{1}}, \cdots, C_{p_{r}}$. Then the expansion (*) can be rewritten as

$$
\begin{aligned}
C_{x} C_{y}= & C_{p_{1}} \cup \cdots \cup C_{p_{r}}=\sum_{i=1}^{r} C_{p_{i}}-\sum_{i<j} C_{p_{i}} C_{p_{j}} \\
& +\sum_{i<j<k} C_{p_{i}} C_{p_{j}} C_{p_{k}}-\cdots
\end{aligned}
$$

Determining any coefficient $\phi_{x y}(p)$ calls for further expanding each term on the right that is not already a cone until ultimately every term is reduced to a linear combination of cones, and then adding over all terms.

In fact, however, it is simpler to determine first the sum of all $\phi_{x y}(q)$ for $q$ in the filter $F_{p}=\{q \in P: q \geqq p\}$ above $p$. Suppose $C_{q_{1}} \cdots C_{q_{t}}$ is any term sooner or later arising in the expansion of $(* *)$, and that its expression as a linear combination of cones is $\sum \pi_{r} C_{r}$. Then the sum of all those $\pi_{r}$ for which $r \in F_{p}$ can be described as the "contribution" of the term $C_{q_{1}} \cdots C_{q_{t}}$ to the sum $\sigma_{x y}(p)=\sum_{g \in F_{p}} \phi_{x y}(q)$.

Lemma 2. If $C_{q_{1}}, \cdots, C_{q_{t}}$ are cones within $C_{x} \cap C_{y}$ then:

(a) if there is any $i$ with $p$ $\sigma_{x y}(p)$ is $\mathbf{0}$;

(b) if $p \leqq q_{i}$ for each $i$ this contribution is 1 .

Proof. If again $C_{q_{1}} \cdots C_{q_{t}}=\sum \pi_{r} C_{r}$ then whenever $\pi_{r} \neq 0$ for some $r \in F_{p}$ it must be that $p \leqq r \leqq q_{i}$ for each $i$.

The proof of (b) is by induction on $h$, the maximum of the heights $h t\left(C_{p}, C_{q_{i}}\right)$ from $C_{p}$ to $C_{q_{i}}$. For $h=0$ the term $C_{q_{1}} \cdots C_{q_{t}}=C_{p}$ does contribute 1 to $\sigma_{x y}(p)$. Assume the lemma true whenever the maximum of these heights is less than $h$ and now suppose that $q \leqq p_{i}$ for 
each $i$ and $\max h t\left(C_{p}, C_{q_{i}}\right)=h$. Notice that if $t=1$ the term is just $C_{p_{1}}$ and hence does contribute 1 to the sum.

Now with $t>1$ and all $h t\left(C_{p}, C_{q_{i}}\right) \leqq h$ any cone $C_{r_{k}}$ which is maximal in $C_{q_{1}} \cap \cdots \cap C_{q_{t}}$ must have $h t\left(C_{p}, C_{r_{k}}\right)<h$ so that for

$$
C_{q_{1}} \cdots C_{q_{t}}=C_{r_{1}} \cup \cdots \cup C_{r_{s}}=\sum_{i=1}^{s} C_{r_{i}}-\sum_{i<j} C_{r_{i}} C_{r_{j}}+\cdots
$$

each term on the right contributes 1 to $\sigma_{x y}(p)$, by induction, and hence the total contribution to the sum from $C_{q_{1}} \cdots C_{q_{t}}$ is just

$$
\left(\begin{array}{l}
t \\
1
\end{array}\right)-\left(\begin{array}{l}
t \\
2
\end{array}\right)+\left(\begin{array}{l}
t \\
3
\end{array}\right)-\cdots+(-1)^{t-1}\left(\begin{array}{l}
t \\
t
\end{array}\right)=1 \text {. }
$$

TheOREM. For any $x, y$ and each $p \in C_{x} \cap C_{y}$ the sum $\sigma_{x y}(p)=1$.

Proof. Suppose $C_{p_{1}}, \cdots, C_{p_{r}}$ are the maximal cones in $C_{x} \cup C_{y}$ with subscripts so chosen that the first $s$ generators $p_{1}, \cdots, p_{s}$ are in the filter $F_{p}$ and the rest are not. The terms $C_{p_{i_{1}}} \cdots C_{p_{i_{k}}}$ of the expansion (**) can be split into two classes according as all $p_{i_{j}} \in F_{p}$ or not. Then (**) gives $C_{x} C_{y}=\sum^{\prime}+\sum^{\prime \prime}$ where each term in the former sum $\left(\sum^{\prime}\right)$ has all $p_{i_{j}} \in F_{p}$ and each term in the latter has at least one $p_{i_{j}} ¥ p$. Now Lemma 2 shows

(a) that the whole contribution to $\sigma_{x y}(p)$ comes from the first sum $\left(\sum^{\prime}\right)$ and

(b) that each term in this sum contributes 1 . But $\sum^{\prime}$ is precisely the same as the expansion by $(*)$ of $C_{p_{1}} \cup \ldots \cup C_{p_{\text {s }}}$ and hence

$$
\sigma_{x y}(p)=\left(\begin{array}{l}
s \\
1
\end{array}\right)-\left(\begin{array}{l}
s \\
2
\end{array}\right)+\cdots+(-1)^{s-1}\left(\begin{array}{l}
s \\
s
\end{array}\right)=1 .
$$

A straightforward Möbius inversion using the $\mu$-function of $P$ (see [1]) now yields a simple formula for $\phi_{y x}(p)$.

Corollary. For each $x, y$ and $p$ in $P: \phi_{x y}(p)=\sum_{q \in P} \mu(p, q) \sigma_{x y}(q)$. Hence the product, $\circ$, defined by cone multiplication is given by $x \circ y$ $=\sum_{p \in P}\left(\sum_{q \in C_{x} \cap c_{y}} \mu(p, q)\right) \cdot p$.

The product takes this form since $\sigma_{x y}(q)=1$ or 0 according as $q \in C_{x} \cap C_{y}$ or not.

When the order on $P=\left\{x_{0}, x_{1}, x_{2}, \cdots\right\}$ can be extended to that of the natural numbers its incidence algebra $a(P)$ (see [1]) can be taken to be upper triangular matrices including the Möbius function $M$ with $m_{i j}=\mu\left(x_{i}, x_{j}\right)$ and its inverse the zeta function $Z\left(z_{i j}=1\right.$ or 0 as $x_{i} \leqq x_{j}$ or not). Representing each $x_{i} \in P$ by the column vector with 
$i$ th component 1 and all others 0 makes $V(P)$ a left $Q(P)=$ module consisting of finitely nonzero vectors $x=\sum_{i} \xi_{i} x_{i}$ and having a convolution, *, given by $\left(\sum_{i} \xi_{i} x_{i}\right) *\left(\sum_{j} \eta_{j} x_{j}\right)=\sum_{i} \sum_{j} \xi_{i} \eta_{j}\left(x_{i} \circ x_{j}\right)$.

CoRollary. If $\cdot$ denotes componentwise multiplication of column vectors, then the product of $P$-elements is given by $x_{i} \circ x_{j}=M\left(Z x_{i} \cdot Z x_{j}\right)$ and hence the convolution $x * y=M(Z x \cdot Z y)$.

Thus the operator $Z$ defines a convolution transform $Z(x * y)$ $=Z x \cdot Z y$, and this extends to order algebras the interesting concepts and applications introduced by Tainiter [4] for finite semigroups.

\section{REFERENCES}

1. G.-C. Rota, On the foundations of combinatorial theory. I: Theory of Möbius functions, Z. Wahrscheinlichkeitstheorie und Verw. Gebiete. 2 (1964), 340-368. MR 30 \#4688.

2. - Möbius function and Euler characteristic, to appear in Festschrift in honor of Richard Rado, Academic Press, New York, 1970.

3. Louis Solomon, The Burnside algebra of a finite group, J. Combinatorial Theory 2 (1967), 603-615. MR 35 \#5528.

4. Melvin Tainiter, Generating functions on idempotent semigroups with applications to combinatorial analysis, J. Combinatorial Theory 5 (1968), 273-288. MR 38 \#65.

University of North Carolina, Chapel Hill, North Carolina 27514 